\title{
COMMISSION 8: POSITIONAL ASTRONOMY (ASTRONOMIE DE POSITION)
}

\author{
President : M. Miyamoto \\ VICE-PRESIDENT : L. V. Morrison \\ Organizing Committee : P. Benevides-Soares, D. P. Duma, L. Helmer, J. A. Hughes, \\ J. Kovalevsky, L. Lindegren, Hu Ning-sheng, F. Noël, G. I. Pinigin, \\ Y. Réquième, H. Schwan, C. A. Smith, M. Yoshizawa
}

\section{Introduction}

Considering the increase of common interests and cooperative activities among related commissions, the commission held four scientific sessions jointly with the Commissions 24,26 , and 40 . These sessions provided worthwhile forums for discussing the common issues such as the global astrometry over the northern and southern hemispheres, and the linking the optical and radio reference frames.

The reporter (now Ex-President) wishes here to thank the commission presidents involved who made these meetings very worthwhile, and to especially acknowledge Prof. W. F. van Altena, the president of Comm. 24, whose contribution was noteworthy. The Joint Discussions of interest to the commission on "Reference Systems : What are they \& What's the Problem ?" (co-sponsored by Comms. 4, 7, $8,19,20,24,31,33$, and 40) and on "HIPPARCOS - an assessment" (co-sponsored by Comms. 4, $8,19,24,31,33,40$, and 44$)$ are reported elsewhere.

\author{
Business Meeting \\ Chairman : M. Miyamoto
}

The first Business Meeting was held on 24 July, 1991. The President welcomed the members of the commission to Buenos Aires and announced the appointement of E. F. Arias as the commission secretary during the XXIst General Assembly. Then, he asked for a moment of silence to pay tribute to the memory of Dr. L. B. F. Clauzet, who had been a very active member of the commission for many years.

1. Organization and Membership

The President announced the results of the election of the commission officers for the coming triennium, and the commission approved unanimously the following officers :

President : L. V. Morrison

Vice-President : C. A. Smith

Organizing Committee : P. Benevides-Soares, T. E. Corbin, L. Helmer, J. Kovalevsky,

L. Lindegren, J. A. López, M. Miyamoto(ex-officio), Hu Ning-sheng,

F. Noël, D. D. Polojentsev, Y. Réquième, H. Schwan, M. Yoshizawa.

The followings were proposed and approved as new members of the commission : W. F. van Altena, Robert W. Argyle, Mireille L. Bougeard, Beatrince Bucciarelli, Ole Einicke, M. Froeschlè, M. Fujishita, Gao Buxi, G. H. Kaplan, K. Kurzynska, Maria Sarasso, Qi Guan-Rong, J. Reynolds, and Laurence G. Taff. Since the XXth General Assembly, four members have resigned from the commission. The total membership is now 160. The President announced that the commission will cosponsor the following two IAU Symposia : 
Developements in Astrometry and their Impact on Astrophysics and Geophysics

(proposers : I. I. Mueller and Ye Shuhua)

Shanghai, China; September 1992.

Sub-arcsecond Radio Astronomy

(proposers : R. D. Davies and R. S. Booth)

Univ. of Manchester, UK; July 1992.

The second short Business Meeting was held on 31 July immediately before the last scientific session chaired by L. V. Morrison. Considering that the commission had not received any report of the activities of the Working Group on Astronomical Refraction and the Working Group on Horizontal Meridian Circle, the continuation of the two working groups was considered. Recognizing the importance of astronomical refraction in ground-based optical and radio astrometry and the need for increased accuracy, the commission decided to set up the following new Working Group on Astronomical Refraction :

J. A. Hughes (chairman), D. G. Currie, A. I. Nefedeva, T. Rafferty, T. A. Th. Spoelstra, M. Yoshizawa.

The commission postponed a final decision on the discontinuation of the WG on H. M. C. till the next General Assembly.

\section{Working Group on Star Lists}

T. E. Corbin gave only a brief report on the present status of the Working Group, because details of the activities were to be presented later at the scientific meeting jointly held with Comm. 24. In view of the universal need for a unified list of fundamental stars fainter than the FK5 Extension, Commissions 8 and 24 formed at the XIXth G. A., the following Working Group on Star Lists :

T. E. Corbin (Chairman), G. Carrasco, L. Helmer, Luo Ding-Jiang, M. Miyamoto, L. V. Morrison,

D. D. Polojentsev, Y. Réquième, and S. Röser.

T. E. Corbin announced that the Working Group would propose the following recommendations at the scientific session on 31 July :

a) that the list of about 3000 stars being prepared by the Working Group be designated as future fundamental stars in the apparent magnitude range 9.5 to 13.0 ,

b) that this list be generally distributed - especially to those who are actively involved in programs to determine star positions,

c) that these stars be observed to relate their positions to the extragalactic system and the dynamical system, in accordance with the recommendations of the Working Group on Reference Systems.

These recommendations were approved at the scientific meeting "Optical Reference Frame and Catalogues - Fainter Extension of the Optical Frame" on 31 July.

\section{Working Group on Astrolabes}

The report on activities of the WG was presented by F. Chollet. After the MERIT Campaign, the astrolabe objectives were shifted to star catalog observations. In order to cope with these activities, the WG was reorganized and the following membership confirmed : F. Chollet (Chairman), V. Gubanov, Li Dong Ming, and F. Noël. In order to increase the quality and scope of the astrolabe, this triennium was devoted to instrumental modification at Santiago (Chile), Sao Paulo (Brazil), San Fernando (Spain), CERGA (France), Paris (France). Some projects to install modern astrolabes are in progress in Turqia and Marocco. The following result; obtained in this triennium should be noted : The photoelectric astrolabe of CERGA has been devoted to the star catalog observation, obtaining very good precision of 0.17 , and the solar astrolabe has continued to observe the solar diameter with a precision not far from 0 ". 2, by using a CCD micrometer. 


\section{Developments in Space and Ground-based \\ Instrumentation, 26 July 1991 \\ Chairman : Y. Réquième}

D. J. Hutter and J. A. Hughes reported on the current status of the design and construction of the U. S. Naval Observatory Astrometric Optical Interferometer (AOI), a dedicated astrometric interferometer observatory which will be in operation at a site in the western U. S. in 1993.

The AOI will be built on the experience gained from the Mark III Optical Interferometer on Mt. Wilson, which was a joint project involving NRL, SAO, USNO, and MIT. The Mark III has already demonstrated wide-angle astrometry with uncertainties of 5 to 10 mas from observations repeated over several nights and systematic agreement with FK5 catalog positions within the larger quoted uncertainties. The new AOI will consist of 4 elements in a fixed array, with baselines arranged for instantaneous position measurement in two dimensions. State-of-the-art delay lines as well as extensive laser metrology will be incorporated. Several beam combination techniques will be used to increase the precision of astrometric measurements. The AOI will achieve unprecedented precision in the optical regime, with statistical and systematic errors below 5 mas and 1 mas for wide angle and narrow angle measurements. This array will establish a catalog of positions of over 1000 stars.

R. C. Stone and D. G. Monet reported on the CCD Transit Telescope (its detector, environmental and laser metrology systems, etc.) under development at the U. S. Naval Observatory Flagstaff Station. The telescope $(20 \mathrm{~cm} \phi)$ uses a CRAF $1024 \times 1024$ (12 micron pixel) cooled chip for scanning regions of the sky and can observe objects with magnitudes between $V=4.0$ to 17.5 . Various scanning techniques are used that enable stars to be scanned from the equator to the pole with little distortion of the images. The telescope can observe about 9000 stars an hour in long strip scans and accurate positions $(\sigma \sim \pm 0.1)$ can be determined for them that are closely tied into the extragalactic VLBI reference frame. Further improvements in accuracy are hoped for by numerical modeling of refraction and the use of laser interferometry to monitor instrumental motions in real time.

L. Helmer reported on the recent implementation of CCD technique on the Carlsberg Automatic Meridian Circle. A new CCD camera, intended for reading the divided circle, has been developed at Copenhagen University Observatory. The camera has been tested in the laboratory on a graduated circle from Heidenhein, and it has been shown that the standard deviation of the reading of one division line is as low as 0.003 , with 1 second integration and evaluation time. This system together with the new circle will be mounted on La Palma in 1992. Furthermore, a more sophisticated CCD camera for detecting star transits is under development, and will be tested in both fixed frame and drift-scan modes on old Cooke Transit Circle now at Copenhagen University Observatory.

M. Yoshizawa presented a progress report on development of a Drift-Scanning CCD Micrometer. Tokyo PMC group has constructed a prototype model of the micrometer "DISC" (DIgital strip Scanning Ccd micrometer), in which the electric charges stored in CCD pixel are successively transferred to the next pixel in order to follow the diurnal motion of celestial objects without any mechanical motion of the micrometer. The prototype DISC consists of a single-field CCD image sensor TH7883 (Thomson-CSF) cooled by liquid nitrogen down to about $170^{\circ} \mathrm{K}, \mathrm{CCD}$ drive board, 16 bit A/D converter, and EWS to control the whole system. The prototype DISC was attached to the Gautier Meridian Circle at Mitaka and tested in the last winter, observing stars and open star clusters with good photometric standards. It was shown that DISC can observe stars down to $V=14.5$ in the rather bright night sky of Mitaka. The construction of a next generation DISC with a larger CCD chip, EEV 88331, has been started as a result of this encouraging experience.

F. Laclare gave a presentation of a newly developed CCD camera to be fitted to the Solar Astrolabe of CERGA, so as to make measurement of the solar diameter more objective. 
Variations of the solar diameter have been detected in a long series of observations made with the solar astrolabe. But, there remains still a suspicion that the cause of the variations may be an observational or atmospheric effect. The results obtained during the two experimental observations with the CCD camera and the Danjon micrometer show very good agreement between the impersonal and visual observations. The dispersion of the daily measurements of the solar diameter with the camera is about 0.1 , which is a significant improvement in quality over visual observations ( 0.18$)$. Moreover, the two dimensional analysis of the digitized solar image should allow the evaluation of a possible atmospheric contribution to the observed variations to be made.

\section{Progress Report on Observing Programs, 26 July 1991 \\ Chairman : L. Helmer}

T. E. Corbin, E. Holdenried, G. Wycoff, and T. Rafferty reported on the current status of the USNO Transit Circles. The 6" Transit Circle in Washington and the $7^{\prime \prime}$ Transit Circle at Black Birch (New Zealand) are both currently in the second circle of the Pole-to-Pole Fundamental Program. The 6" TC started its second circle in Feb.1990 and to date 53457 observations have been made. Combined with the first circle results this makes a total of 175511 observations on the $6^{\prime \prime} \mathrm{TC}$ thus far in the program. The total for the first circle of the $7^{\prime \prime} \mathrm{TC}$ is 156472 observations, and the second circle was begun in May 1991. The $7^{\prime \prime}$ TC has had particular success with its observations of day stars with 18330 made during the first circle. It appears possible to reduce and model the 7" TC day observations separately from the night, thereby giving a much stronger solution for the observations of the Sun, Mercury and Venus.

Comparisons of the absolute observations with the FK5 generally show the same features as found by the CAMC, Bordeaux AMC, and Tokyo PMC programs north of $-20^{\circ}$. South of that declination there is weak oscillating feature in right ascension going to about $-50^{\circ}$ and a strong positive trend, in the sense of Black Birch - FK5, south of there. The declination differences mainly show a strong positive feature from $-30^{\circ}$ to $-65^{\circ}$.

L. V. Morrison reported on CAMC observations of solar system objects. The CAMC spends about $2 \%$ of its time on observing solar system objects. Since the beginning of observing on La Palma the CAMC has made a total of about 13000 observations of Mars, Callisto, Saturn, Titan, Iapetus, Uranus, Neptune, Pluto and 60 minor planets. The accuracy of an observation ranges between 0.14 and $0 . ' 25$ depending on the zenith distance and magnitude, as described in the Introductions to the Carlsberg Meridian Catalogs.

A detailed analysis of the Titan and Iapetus observations combined with those made at Bordeaux produce very consistent opposition corrections to JPL DE202 for Saturn for the years 1987-89 (D. B. Taylor et al., 1991). These corrections are much more reliable than those derived from direct observations of Saturn's disk. An analysis of the CAMC observations of Pluto (the first ever made by a meridian circle) gives corrections of +0.4 and +0.2 to R.A. and Dec. of JPL DE202, respectvely. These are consistent with Klemola's astrographic results of 1988 and Mink's occultation result of 1988.

M. Yoshizawa and M. Miyamoto gave a progress report on the First Tokyo PMC Program which started in 1985 and is to end in 1992. The Program comprises about 33000 stars and the solar system objects (the Sun, 6 major planets, Ceres, Pallas, Juno, Vesta, Hebe, Iris, Flora, Metis, and Eunomia). The program stars are composed of FK5 stars, NPZT stars, AGK3RN stars, Zodiacal stars, OB stars, $\mathrm{H}_{2} \mathrm{O}$ masers, and faint reference stars around QSO's. The three annual catalogs, Tokyo PMC 85 (1007 stars), 86 (3974 stars), and 87 ( 5748 stars) have been published. The fourth catalog, Tokyo PMC 88 ( 3845 stars) will be published soon. A local systematic error of about 0.1 in the FK5 system is evident in Tokyo PMC 86 and 87, suggesting an erroneous proper motion system of the FK5 in some parts of the sky. The final goal of the First Tokyo PMC Program is to provide absolute positions of the program stars, consistent with the dynamical theory of the planetary system. The compilation of the absolute Tokyo PMC catalog will start in 1992 . 
S. Sadžakov, M. Dačić, and Z. Cvetković reviewed the recent observations of solar system objects (the Sun, Mercury, Venus, and Mars) performed with the Large Meridian Circle of Belgrade Astronomical Observatory, and gave an error estimate of these observations. They stressed that the quality of the Belgrade observations with regard to both systematic and random errors is comparable to other modern observations.

A paper by D. D. Polojentsev and R. Zalles was read by V. Abalakin. The paper reviewed the current astrometric activity with the astrograph $\left(D=23 \mathrm{~cm}, F=230 \mathrm{~cm}\right.$, field $4^{\circ} \times 4^{\circ}$, scale $\left.90^{\prime \prime} / \mathrm{mm}\right)$ in the Soviet-Bolivian Astronomical Observatory at Tarija. The observing programs being carried out are : FOKAT-S Catalog to provide precise positions of about 200,000 southern stars up to 11th magnitude, Bright Star Catalog to supplement bright southern stars not included in the FK4 catalog, and Equatorial Star Catalog to provide reference stars with density of 40 stars per square degree.

Another paper by A. S. Kharin was also read. The paper presented a list of 836 FK5 stars observed in the infrared range. These stars will be used as reference stars in IR range with positions and proper motions given in the FK5 system. The diskette of the list will be provided on request.

\author{
Astrometry in the Southern Hemisphere \\ (joint with Comms. 24 and 26), 27 July 1991 \\ Chairman : J. A. López
}

The activities of this session are described by W. F. van Altena in the Report of Commission 24 .

\author{
Linking Optical and Radio Reference Frames \\ (joint with Comms. 24 and 40), 29 July 1991 \\ Chairman : T. E. Corbin
}

K. Johnston reviewed the present status of linking the optical and radio reference frames. The radio reference frame appears to be well defined at the mas level in the northern hemisphere and down to a declination of $-30^{\circ}$. Observations initiated over the past two years for sources south of $-30^{\circ}$ have resulted in accuracies of order ten mas level (Russell). The initiation of dual frequency S/X VLBI observation in the southern hemisphere will result in positional accuracies at the mas level for a large number of sources by 1993. Observations for a global radio reference frame of 400 sources are being carried out (Russell) to extend the present number of reference sources obtained through geodetic programs. The optical reference frame now in use is the FK5 which is believed to be accurate to 50 mas level with zonal errors in the southern hemisphere sometimes approaching a hundred mas.

There are three methods being followed to relate these two frames. The first method is to determine the optical positions of the extragalactic radio sources making up the radio frame (Johnston, Russell, de Vegt). The optical positions of the radio sources can be measured relative to nearby reference stars with a precision at the 10 mas level on prime focus plates obtained with a $4 \mathrm{~m}$ class telescope. These reference stars can be related to FK5 stars by wide field astrograph plates. The accuracy of the position of the radio sources on the FK5 frame is estimated to be at the 50 mas level (de Vegt). The second method is to determine the positions of bright stars which also display radio emission (Lestrade, de Vegt). Many modern transit circle programs are being undertaken to refine the positions of these stars on the FK5 system. Meanwhile, VLA and VLBI observations of about fifty stars which emit continuum radiation have been made (Johnston). For stars emitting continuum radiation, it is probable that the optical and radio centers coincide at a few mas level. Preliminary comparison of the optical and radio observations shows that the reference frames in the northern hemisphere coincide at the 100 mas level when appropriate zero point alignments and rotations are made. In the third method, bright stars which display maser emission are used to relate the reference frames. Sufficient observations have to be made to relate the photocenter to the circumstellar shell emitting maser radiation. The masers which are located closest 
to the stellar photosphere, such as the $\mathrm{SiO}$ masers, may be capable of relative accuracies at the 10 mas level; but this has still to be demonstrated.

Although the present accuracies of the frame-tie measurements are limited to the 50 mas level (cf. FK5) the success of the HIPPARCOS mission will improve the accuracy of the optical frame to a few mas level. With the advent of this improved accuracy, future frame-tie observations must be made with mas accuracy to relate the both frames.

R. C. Stone gave a description of the use of a CCD Transit Telescope for linking reference frames and presented preliminary observational results obtained with it. The U. S. Naval Observatory at Flagstaff has developed a CCD transit telescope capable of observing both FK5 stars as well as extragalactic sources defining the radio reference frame. Objects as faint as $V=17.5$ can be observed. With a screen placed in front of the objective, the bright limit is extended to $V=3.5$. The accuracy of the position in both coordinates is about 0.1 . Thus, observations of FK5 stars and VLBI extragalactic sources can be observed directly with this instrument, and reductions can be made on a nightly basis. The transit circle thus developed can be used for determining the offsets between the optical and radio positions of extragalactic objects, and can determine accurate positions for a large numbers of faint stars that are tied into the extragalactic radio reference frame.

An analysis of the positions of VLBI extragalactic sources obtained with the instrument indicates that some sources have large differences between their radio and optical positions. Observations of radio stars and FK5 stars indicate that a difference between the VLBI and FK5 reference frames can exceed 0."1, but a better agreement of both frames is found for the radio stars. These preliminary results will be improved by further observation.

M. Yoshizawa and S. Suzuki presented the optical position of $3 \mathrm{C} 273 \mathrm{~B}$ determined by a CCD meridian circle. A prototype model of the CCD meridian circle developed at National Astronomical Observatory of Japan was used to determine directly the optical position of $3 \mathrm{C} 273 \mathrm{~B}$ on the FK5 system. The right ascensions of 3C273B and faint stars around it were determined with "DISC" (DIgital strip Scanning $\mathrm{Ccd}$ micrometer) fitted to the Gautier meridian circle at Mitaka. The observed position of $3 \mathrm{C} 273 \mathrm{~B}$ was found to coincide with its best available radio position (R.A.) to within 0.07 .

L. V. Morrison reported on the photographic astrometry of the four radio stars, Z Her, 9 Sgr, HD193793, and SV Cam (R. W. Argyle et al., 1991), carried out in a test program using the Wide Field Camera (f/8) of the Jacobus Kapteyn Telescope in conjunction with the automatic plate measuring machine at Cambridge. In all cases the positions of the reference stars used were provided by the Carlsberg Automatic Meridian Circle catalogs. From this test program an accuracy of about 0."04 was obtained for the optical positions of radio stars.

It was found that there is no detectable magnitude equation even though target and reference stars differ by up to $5 \mathrm{mag}$. Thus, by observing the reference stars directly with the CAMC there is no need to construct a secondary frame of reference to connect the radio stars to the FK5 system. This encouraging result has led to the expansion of the test program to include all radio stars with positions measured by the VLA, directly by VLBI and in progress with MERLIN. The aim of the expanded program is two-fold : to link the optical reference frame defined by the FK5 to the VLBI radio frame and to link the HIPPARCOS frame similarly.

C. Fabricius gave a progress report on the CAMC program for linking the optical and radio reference frames. Nine QSO's with VLBI radio positions are being observed. Furthermore, 9300 reference stars of $V \sim 11$ in 360 fields have already been observed. A new observing list of fainter stars of $12 \leq V \leq 14$ within a field of $30^{\prime} \times 30^{\prime}$ around the $400 \mathrm{VLBI}$ sources is being prepared. This list will be suitable for a wide field CCD micrometer. 
Y. Réquième reported on the Bordeaux Observations of radio stars. Optical positions of 221 radio stars have been obtained with the Bordeaux Automatic Meridian Circle in the period of 1984 to 1990 (Réquième and Mazurier, 1991). The observations were strictly differential, with 6 to 8 FK5 stars per hour in the range of $-25^{\circ}<\delta<+75^{\circ}$ observed in order to determine the 8 instrumental parameters. The mean positions given at $\mathrm{J} 2000$ were corrected for chromatic refraction and also for photocenter effect in the case of duplicity. The expected accuracy is about 0.05 at least for the northern stars which were observed 32 times on the average. The mean position of $3 \mathrm{C} 273 \mathrm{~B}$ was also obtained from 35 observations under the same conditions.

P. Hemenway and N. Argue reported on the HIPPARCOS Link Plans. Assuming that the VLBI extragalactic reference frame represents a close approximation to an inertial frame, the HIPPARCOS Project has been pursuing observations to link the HIPPARCOS frame to the VLBI frame in both position and rotation. Since HIPPARCOS cannot observe extragalactic objects directly, two indirect approaches are being taken. First, the positions and rotations of about 40 radio stars included in HIPPARCOS are being observed with the VLA and VLBI. Second, optical counterparts of VLBI radio sources are being observed optically with respect to the HIPPARCOS frame. The highest accuracy observations will be made of a few tens of sources with the Hubble Space Telescope and ground-based CCD devices. Furthermore, several hundred sources will be observed photographically, sometimes combined with CCD observations. The final link accuracy is expected to be between 0.002 and 0.0001 in positional offset, and 0."002 /yr and $0.0001 / \mathrm{yr}$ in rotation.

R. J. Davis stressed the importance of observations of radio stars with "MERLIN" at an intermediate resolution for linking the optical and radio reference frames. The radio stars are not always point sources at radio frequencies and the stellar radio centroid depends on the resolution of the interferometer system used. It is known that thermal and non-thermal components exist in these stars and these components may be spatially separated and related to material in different orbits around the central star. The VLBI is sensitive only to the non-thermal component, while the MERLIN with its resolution intermediate between the VLA and VLBI retains surface brightness sensitivity to detect the thermal as well as the non-thermal emission. The intermediate resolution will therefore play an important part in establishing the location of the emission as a function of orbital phase and during any radio/optical outbursts. Thus, the MERLIN link observations should give the global rotation matrix needed to bring the optical frame into alignment with the VLBI frame.

\section{Progress Reports on Catalogs, 29 July 1991 Chairman : C. Smith}

M. Yoshizawa and S. Suzuki presented the third and fourth annual catalogs of the Tokyo PMC 87 and 88 of 5748 and 3845 stars, respectively. The mean systematic trends of the observed positions minus those of FK5 basic stars agree quite well with each other for all the Tokyo PMC catalogs. It is confirmed that the FK5 system has a systematic error $\Delta \delta_{\delta}$ of about $0^{\prime \prime} 1$ in the declination zone $40^{\circ}<\delta<60^{\circ}$. The systematic error must be explained by an imperfection in the FK5 proper motion system.

C. Fabricius reported on the Carlsberg Meridian Catalogs No.5-No.7, which are based on the observations with the new moving slit micrometer fitted in May 1988. Catalogs Nos. 5 and 6 have yielded the positions of about 35000 stars with a zenith mean error of 0.08 in R. A. and Dec., and 0." 04 in magnitude. The proper motions of these stars have been derived by combining the positions with these in catalogs at earlier epochs. The mean errors of the proper motions are between 0."002 / year and 0."003 / year. The Carlsberg Meridian Catalogs (Nos. 1-7) constitute a reference net with a density of 2.5 stars per square degree.

R. A. Carestia and C. C. Mallamaci proposed an alternative format for presenting positions and proper 
motions of the FK5 stars in equatorial rectangular coordinates.

G. Carrascco and P. Loyola reported on the results of differential observations of the FK5 stars, carried out with Repsold Meridian Circle at Cerro Calán National Observatory. A total of 10153 observations were obtained for the declination zone $+40^{\circ}$ to $-90^{\circ}$. The mean observational epoch is 1988.3 and the mean internal error of the positions is $\pm 0 . " 08$.

M. Yoshizawa and M. Miyamoto reported on the optical positions of $51 \mathrm{H}_{2} \mathrm{O}$ maser sources (mainly Miratype variables), observed in 1987 with Tokyo PMC. The observed sources are distributed on the sky rather uniformly, which is benefited for the global link of the optical and radio reference frames. The positions of a set of about $60 \mathrm{H}_{2} \mathrm{O}$ maser sources were also observed with VLA in 1986. Fifteen of those sources are common to these observed to the Tokyo PMC. The optical and radio positions of these common sources are being compared.

E. F. Arias reviewed on the IERS Celestial Reference Frame (IERS CRF). The IERS is responsible for defining and maintaining a celestial reference frame based on the positions of extragalactic radio sources with VLBI.

The first realization of the IERS CRF (Arias et al., 1988) fixed the initial definition of axes of the frame. Each year, as new individual realizations are submitted to IERS, a new version is computed. The IERS Ox axis is in agreement with the FK5 origin of R.A. The $\mathrm{Oz}$ axis points in the direction of the mean pole at $\mathrm{J} 2000.0$ as defined by the conventional models for precession and nutation. The successive realizations of IERS CRF elaborated up to now have maintained the initial definition of the axes within

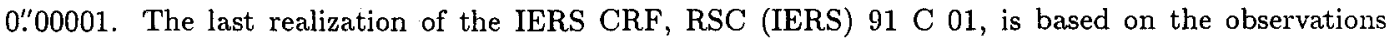
of a total number of 396 compact extragalactic radio sources uniformly distributed on the sky in the range $-83^{\circ}<\delta<+85^{\circ}$. These VLBI data have been compiled on the basis of two global solutions independently obtained by GSFC and JPL. The axes of RSC (IERS) $91 \mathrm{C} 01$ are fixed by 57 primary sources selected by statistical tests.

\section{Optical Reference Frame and Catalogues - Fainter Extension of the Optical Frame - (joint with Comm. 24), 31 July 1991 \\ Chairman : K. Johnston}

T. E. Corbin and H. Schwan reviewed the extension to the FK5 catalog. Work on the "Basic FK5" giving the classical fundamental stars has been completed and the printed version published. In the second part of the FK5 (the "FK5 Extension"), improved positions and proper motions for 3117 new fundamental stars will be given. There are 992 stars selected from the FK4 Sup catalog and 2125 stars from the IRS list, extending the fundamental system to about $V=9.5$. The determination of the mean positions and proper motions for the new fundamental stars has been completed and the compilation of various auxiliary data on a uniform basis, such as parallaxes or apparent magnitudes, has been finished.

The following data represent the characteristics of the FK5 Extension :

Average mean epoch : 1944

Average mean error of mean positions at mean epoch : 0.055

Average mean error of proper motions : $0.255 / \mathrm{cy}$

The final catalog is given in the system of the Basic FK5 and based on the IAU (1976) System of Astronomical Constants. The format is, as far as possible, the same as for the Basic FK5. A tape version of the catalog was sent to the astronomical data centers in June 1991.

T. E. Corbin and L. V. Morrison gave a progress report from the Working Group on Star Lists, concerning the Intermediate Fundamentals (IF) - a unified list of fundamental stars fainter than the FK5 Extension. The Working Group has discussed and approved that a list of about 3000 stars in the magnitude range $9^{\mathrm{m}} 5$ to $13^{\mathrm{m}} 0$ with roughly equal numbers of stars from $9^{\mathrm{m}} 5$ to $11^{\mathrm{m}} \cdot 0$ and $11^{\mathrm{Im}} 5$ to $13^{\mathrm{m}} \cdot 0$ should 
be selected. It was further agreed that first consideration should be given to stars in the HIPPARCOS Input Catalog and to those included in current observing programs such as the CAMC reference stars near extragalactic benchmark radio sources.

The IF1 list ( $9^{m} 5$ to $11^{\mathrm{m}} 0$ list) was compiled by L. V. Morrison, resulting in 1672 stars showing an even distribution over the sky, while the IF2 list $\left(11^{\mathrm{m}} 0\right.$ to $13^{\mathrm{m}} 0$ list $)$ was selected by T. E. Corbin. The total number of the IF2 stars amounts to 1143, composed of 10 HIPPARCOS High Priority Radio Stars, 11 HST-HIPPARCOS Link Stars, 1032 HIPPARCOS Input Stars, and 90 CAMC Reference Stars around benchmark radio sources. Known double stars, variable stars, and high proper motion stars were eliminated from the above lists.

There remain, at this time, several gaps in the distribution of the stars. These gaps cannot be filled with stars from the above-mentioned sources. The Working Group will fill the gaps in the southern hemisphere with stars from CPC2 / FOKAT-S, and those in the northern hemisphere with stars from USNO Astrograph plates, and add other recommended stars such as O.B stars proposed by M. Miyamoto.

After Corbin's presentation and some discussions, the recommendations announced at Business Meeting on 24 July were approved by Commissions 8 and 24 .

S. Röser and U. Bastian presented a reference star catalog of positions and proper motions (PPM) of about 360000 stars covering the whole sky. The PPM is produced at Astronomisches Rechen-Institut, the southern part in cooperation with 3 institutes in the USSR : Pulkovo observatory, Kiev observatory and Sternberg institute. At present the status of the PPM is the following. The northern hemisphere portion was completed in 1989; a tape version is available through international astronomical data centers and a printed version appeared in April 1991. A preliminary version of the southern hemisphere part was completed in mid-1990 and its tape version is also available through the data centers. The final version will be completed at the end of 1991 . The main characteristics of the PPM at present are the following:

\begin{tabular}{|c|c|c|c|c|c|}
\hline & \multirow[t]{2}{*}{ Stars } & \multicolumn{2}{|c|}{ Mean error of position } & \multicolumn{2}{|c|}{ Mean error of p.m. } \\
\hline & & \multicolumn{2}{|c|}{$\begin{array}{l}\text { R.A. } \quad \text { Dec. } \\
\text { at epoch } 1990(\operatorname{arcsec})\end{array}$} & \multicolumn{2}{|c|}{$\begin{array}{l}\text { R.A. Dec. } \\
(\operatorname{arcsec} / \text { cy })\end{array}$} \\
\hline PPM North & 181731 & 0.27 & 0.27 & 0.43 & 0.42 \\
\hline PPM South (Pr.) & 144787 & 0.26 & 0.26 & 0.55 & 0.55 \\
\hline PPM South (4.91) & 144787 & 0.12 & 0.12 & 0.40 & 0.40 \\
\hline \multicolumn{6}{|c|}{ Expected properties of the final PPM South : } \\
\hline PPM South & 180000 & 0.12 & 0.12 & 0.30 & 0.30 \\
\hline
\end{tabular}

Since the summer of 1990 a new reference catalogue for the whole sky has been available which surpasses the SAO catalogue's accuracy by a factor of 3 to 6 in positions and proper motions. For the time being, a version of PPM on the old system of B1950 FK4 is also supplied for observers to take advantage of its higher precision compared to SAOC. The final version of the southern part will contain some 40000 stars more than the preliminary one. This is especially important in the regions $0^{\circ}<\delta<-20^{\circ}$, and south of $-64^{\circ}$. There the density of stars per sq. deg. will be increased from about 5 and 3 , respectively, in SAOC to about 9 in the final PPM South.

T. E. Corbin and S. Urban reported on the compilation of the catalogs of the International Reference Stars (IRS) and the Astrographic Catalog Reference Stars (ACRS), both of which are now available at the international astronomical data centers.

The IRS is based on the AGK3R and SRS lists. The AGK3R portion has been completed for some time, and the recent completion of the SRS has made it possible to complete the IRS. The southern IRS was compiled from 87137 star positions in 94 catalogs, resulting in 19827 mean positions and proper motions. The combination of the southern and northern portions has produced the IRS of 36027 mean 
positions and proper motions with an average epoch of 1949.5. The IRS is divided into two parts : Part I contains the stars with good observational histories and has 29163 stars with average proper motion mean errors of $0 . " 43$ /cy in R.A. and 0." 42 /cy in Dec. The catalog is available on B1950 FK4 and J2000 FK5.

The ACRS is the result of combining data from photographic and meridian circle catalogs in order to give the best proper motions at the highest density possible. The basic lists are the AGK3 in the north and the CPC2 in the south. A total of 1643783 catalog positions from 167 catalogs were reduced with the combined FK4, improved FK4 Sup and IRS to produce 320211 mean positions and proper motions with an average epoch of 1949.5. The ACRS is also divided into two parts : Part I contains 250052 stars with average proper motion mean errors of 0." 47 /cy in R.A. and 0." 46 /cy in Dec. Part II, like that of the IRS, is mostly stars whose proper motions are derived from only two catalog positions. The positions in the catalog are given for both B1950 FK4 and J2000 FK5.

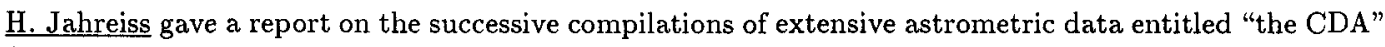
(Catalogue de Donnees Astrometrique), in which the "best" astrometric parameters and cross identifications are given for every star. The first version of the CDA was created in 1984 out of the need to get a quick overview of existing astrometric catalogs fulfilling the required positional accuracy of 1.15 at the epoch of 1990 for the HIPPARCOS satellite. The second version of the CDA compiled in 1988 took into account the individual errors of positions and proper motions of the stars. This version contains positions for 463615 stars and proper motions for 327477 stars. The third version of the CDA has recently been started with the intention to compile astrometric data for as many stars as possible. In particular, stars of astrophysical interest (nearby stars, high proper motion stars, etc.) will be added. At present the CDA contains 466072 positions and 337478 proper motions, providing a mean positional accuracy of 1 " at the epoch of 2000.0 (except 555 stars with large errors). The content of the CDA will soon increase to about 550000 stars when the HIPPARCOS Input Catalog is released.

W. R. Dick reported on the optical work at Bonn, Potsdam and Tautenburg, concerning the linkage of the HIPPARCOS system to the extragalactic objects. Besides the link of the HIPPARCOS reference frame to extragalactic objects via radio stars or by the HST, photographic astrometry is also able to calibrate the HIPPARCOS proper motions with respect to an inertial system. Numerical simulations have shown that even with a very small number of well-distributed link fields, the photographic method is competitive with other techniques ( $\mathrm{P}$. Brosche et al., 1991).

Therefore, the Bonn and Potsdam astrometric groups are engaged in programmes for the HIPPARCOS extragalactic link with ground-based observations. The Bonn programme is based on plates from different telescopes with an epoch difference of up to 90 years. Proper motions have been derived for 45 HIPPARCOS stars around 4 bright QSO's, together with "fictitious" proper motions of the QSO's. The Potsdam / Tautenburg programme uses exclusively plates from the Tautenburg Schmidt Telescope. Measurements of 19 proper motion fields are now complete. On the basis of these recent results, an accuracy of up to $0.1 /$ cy has been estimated for both programmes. Additionally, the data from proper motion studies of globular clusters can be used for the HIPPARCOS extragalactic link. Both at Bonn and Potsdam optical positions of extragalactic radio sources have been derived for the radio / optical link. The external random error is about 0.15 to 0.20 . Improvements to reduction techniques have been also proposed.

$$
\begin{gathered}
\text { Optical Reference Frame and Catalogues - Fainter Extension } \\
\text { of the Optical Frame - (joint with Comm. 24), } 31 \text { July } 1991 \\
\text { Chairman : L. V. Morrison }
\end{gathered}
$$

The activities of this session are described by W. F. van Altena in the Report of Commission 24 . 